Memory Function and the Hippocampus

Bertram Opitz

Department of Psychology, University of Surrey, UK

Short Title: Hippocampus and Memory

Abstract:

There has been a long tradition in memory research adopting the view of a vital role of the medial temporal lobe and especially the hippocampus in declarative memory. Despite the broad support for this notion, there is an on-going debate about what computations are performed by the different substructures. The present chapter summarises several accounts of hippocampal functions in terms of the cognitive processes subserved by these structures, the information processed and the underlying neural operations. Firstly, the value of the distinction between recollection and familiarity for the understanding of the role the hippocampus plays in memory is discussed. Then multiple lines of evidence for the role of the hippocampus in memory are considered. Cumulating evidence suggests that the hippocampus fosters the binding of disparate cortical representations of items and their spatio-temporal context into a coherent representation by means of a sparse conjunctive neural coding. This association of item and context will then lead to the phenomenological experience of recollection. In contrast, surrounding cortical areas have broader neural coding that provide a scalar signal of the similarity between two inputs (e.g., between the encoding and the retrieval). By this they form the basis of a feeling of familiarity but also might encode the commonalities between these different inputs. However, a more complete picture of the importance of the hippocampus for declarative memories could only be drawn when the interactions of the medial temporal lobe with other brain areas are also taken into account.

\title{
Bertram Opitz
}

Department of Psychology

University of Surrey

Guildford

GU2 7XH

UK

phone: +44 1483689449

fax: $\quad+441483689553$

email: b.opitz@surrey.ac.uk 


\section{Introduction}

Ever since the first report of profound amnesia following medial-temporal lobe (MTL) resection in patient H.M. [1], there has been a large amount of research aiming at the functional role of the MTL sub regions, especially of the hippocampus, in memory. This research includes all currently available methods, including neuroimaging studies and electrophysiological recordings in humans, single cell recordings in animals and neuropsychological studies of patients with brain injuries or of animals with experimental lesions. Despite that any research method has its own strength and limitations they all converge on the view that the hippocampus operates in the service of declarative memory. One cognitive account assumes that the MTL is involved in the recognition of a previously encountered event [2-4]. Despite the broad support for this notion, there is an on-going debate about what computations are performed by different sub regions within the MTL. One prominent view capitalizing on bidirectional interconnections between the hippocampus and the surrounding medial-temporal lobe cortex (MTLC) proposes that the hippocampus is important for all forms of declarative memory, including recognition memory [2]. A contrasting view emphasizes the differences between the same structures within the MTL suggesting that the hippocampus and the MTLC support different aspects of recognition memory[3; 5; 6]. In particular, the hippocampus and the parahippocampal cortex were assumed to support recollection, i.e. recognition of an item on the basis of the retrieval of specific contextual details of the previous learning experience, whereas the perirhinal cortex subserves familiarity, i.e. item recognition on the basis of a scalar memory strength but without retrieval of any specific detail about the study episode. These models, however, are hard to reconcile with the notion of a highly integrated network connecting all MTLC structures with each other and, most importantly, with the hippocampus [7]. Thus, more recent modifications sought to overcome the explanatory limitations of models associating MTLC and hippocampal functions in terms of the purely cognitive dichotomy between familiarity and recollection, respectively, by focussing on the kind of information, i.e. item-specific and contextual information, stored by the different substructures of the MTL $[3 ; 8 ; 9]$. Other models emphasize the putative operational characteristics of specific brain regions to describe the role of these regions in memory [7; 10]. The present chapter reviews evidence from animal research, neuropsychological studies with patients suffering from amnesia and the growing body of neuroimaging studies that form the basis of each of the different accounts of the role of the hippocampus in memory.

\section{Cognitive Processes Accounts}

Following a widely acknowledged cognitive view it has been argued that the hippocampus is vital for recognition based on recollection but not for recognition based on familiarity (Figure 1A). These models usually further argue that MTLC regions (especially the perirhinal cortex but not the parahippocampal cortex) are essential for familiarity based recognition, and that this function is independent of the hippocampus [3; 11]. Consistent with this view, patients with severe hypoxic damage to the hippocampus exhibit disproportional large deficits in associative recognition (thought 
to rely on recollection) as compared to simple item memory (relying on familiarity, e.g. [12]). An almost identical pattern of impaired recollection and preserved familiarity has also been observed in a patient with selective hippocampal atrophy caused by meningitis [13]. Most striking evidence in favour of dual process models comes from a double dissociation between deficits in recollection or associative memory following hippocampal damage compared to deficits in familiarity or item memory following damage to the perirhinal cortex [14]. These observations are paralleled by several animal studies demonstrating that rats, initially trained to recognize associations between an odour (e.g. , cumin, lemon, thyme) and one of several digging media (e.g. , sand, wood chips, etc.) and then retested after selective damage to the hippocampus, exhibited impaired recollection of the associations while memory for the odours alone was spared indicating intact familiarity [15]. In the same vein, neuroimaging studies in healthy participants employing associative recognition memory task demonstrated greater hippocampal activity for successful as compared to failed source recollection [16]. In this experiment participants studied a word list while alternating between a pleasant/unpleasant decision and a concrete/abstract decision. At test, they were required to discriminate between two simultaneously presented test words by selecting the member of the pair previously associated with a particular encoding task. Successful source retrieval was associated with increased activity in the left hippocampus.

Another method capitalizes on Receiver-Operating-Characteristics (ROC) assuming that recollection is a threshold process whereas familiarity varies in a continuous manner with response confidence [17]. A number of studies have, therefore, used linear and curvi-linear approximations of confidence ratings (representing recollection and familiarity, respectively) to identify regions where hemodynamic activity systematically varies with recognition confidence [18-20]. Such parametric analyses consistently showed that hippocampal activity was related to recollection. While some studies found increasing activity in the perirhinal cortex as perceived strength of familiarity increased [18], others reported monotonic decreases in activity with increasing memory strength not only in the perirhinal cortex but also in the anterior hippocampus [19]. Yet another result was reported in a recent study [20] observing both decreasing and increasing activity as a function of increasing familiarity in the anterior and posterior perirhinal cortex, respectively. This latter finding emphasizes the contradictory results with respect to the role the MTLC plays in recognition memory.

A prominent alternative view explains the functional distinction between the hippocampus and the adjacent MTLC structures described above with a single cognitive process in which differences in memory strength account for the differential involvement of the hippocampus and the perirhinal and parahippocampal cortices [2; 4]. While a weak memory trace seems sufficient to engage the MTLC, strong memories are required to engage the more powerful computational properties of the hippocampus. Evidence for this view is for example provided by studies demonstrating that amnesic patients are similarly impaired in all kinds of declarative memory [21]. However, single process models cannot account for the double dissociations in amnesia and neuroimaging studies cited above, nor can single process theories account for the double dissociations between the role of the hippocampus and the surrounding MTLC in recollection and familiarity in recent animal studies. 


\section{Information Based Accounts}

More recent models move beyond the simple and rather phenomenological dichotomy between recollection and familiarity towards an understanding of MTL functions in terms of the information they store. As depicted in Figure $1 \mathrm{~B}$ these models propose that the perirhinal and the parahippocampal cortex support the encoding and retrieval of item-specific and contextual information, respectively whereas the hippocampus stores representations of item-context associations [3; 8; 9]. This view is based on increased hippocampal activity in tasks emphasizing such conjunctive memory representations such as memorizing paired associates [22], source memory tasks [23] and tasks requiring also the spatial location of a previously presented item to be remembered [24]. Conversely, in many of those studies activity in the perirhinal cortex correlates with item rather than conjunctive memory performance [22; 24]. Together these studies demonstrated that an increase of activity of the hippocampus is essential for the process of relating an item to contextual information during retrieval. This notion has gained further support from neuropsychological studies in amnesic patients $[25 ; 26]$. For instance, it was demonstrated that amnesic patients could well discriminate between old and new visual scenes but were unable to distinguish between intact old scenes and manipulated old scenes (e.g. by left- right shifting of particular elements within the scene) [26] indicating a deficit in processing the relations of items within a specific context rather than a deficit in recollection, per se.

Parallel evidence has also been obtained from animal studies showing that hippocampal neurons develop representations of the specific combination of stimulus elements (odours $A$ and $B$ ) and the context (room $X$ and $Y$ ) in which they occur [27]. In the beginning of their training the rats' hippocampal neurons responded selectively to a specific location in environment occupied by the animals (so-called "place cells"). However, after several exposures to the same contextual discrimination problem, i.e. odour $A$ is only rewarded in room $X$, when the animals acquired the item-context associations, some neurons began to fire selectively during the sampling of a specific item in a particular context and these cells continued to exhibit item-context specificity after learning. Similar firing patters for the combination of specific stimuli with a location or behavioural context in which they occurred was also demonstrated for monkeys [28] and humans [29]. These results indicate that hippocampal firing patterns reflect unique conjunctions of stimuli with the places and contexts in which the stimuli occur. Extending this view it was proposed that also associations of multiple items that share their cortical representations due to a substantial feature overlap (within the same domain, e.g. two faces or two words) can be stored by the perirhinal cortex and recognized based on their familiarity [30]. Evidence for this assumption comes from several neuropsychological patients with selective hippocampal damage demonstrating severe impairments in the recognition of e.g. object-location and face-voice associations, while they were relatively unimpaired at recognizing pairs of words, non-words, unknown and famous faces after one or several study trials [31].

Common to all the examples described above and more general to typical episodic memory tasks, the item presented at the time of learning has to be associated with its specific study context. Later 
during recognition this association must be retrieved. As argued above the hippocampus enables the retrieval of the association of an item with its study context and, consequently, will lead to the phenomenological experience of recollection. In contrast, the proposed role of perirhinal cortex in retrieving item information alone is consistent view the dual process view of familiarity based recognition. Thus, recollection and familiarity can be regarded as rather epiphenomenal to the information processed within the hippocampus and the MTLC, respectively.

\section{Neural Process Based Accounts}

In a similar vein, others have proposed that functional differences between MTL sub-regions are based on their key computational role in memory [7; 10;32]. Despite their differences these views about MTL functions converge on the opinion that the distinct properties of hippocampal neurons and neurons in the surrounding MTLC subserve different memory processes (Figure 1C). It was suggested that sparse neural coding within the hippocampus will (a) foster the convergence of disparate cortical representations of items, actions, etc. and their spatiotemporal context that compose a unique input into a bound representation of that input and will (b) reduce the probability that the same neurons within the hippocampus are activated by two different inputs, thereby leading to distinct (pattern-separated) representations [10; 32]. The process of binding mentioned in (a) can be specified in terms of relational operations (e.g. identity, greater than or earlier than) that link together and organize the individual elements of an experience. For example, during paired associate learning two items provide relational information about their identity with respect to their spatio-temporal context that is processed by the hippocampus. It is capable to organize any arbitrary relations and by this very effortful but highly flexible. Consequently, it allows for the rearrangement of the elements of individual experiences to deal with novel situations. Crucially, the hippocampal circuitry possesses anatomical and computational characteristics to support these properties of separated relational bindings (see [3], for a detailed discussion). Due to this separation of different inputs mentioned in (b) the hippocampus is able to entirely reconstruct each single input (pattern completion), e.g. an item bound to its study context. It thereby enables the retrieval of contextual information. Thus, the process of relational binding will lead to recognition based on recollection. This close connection between relational binding and recollection was corroborated by animal studies and neuroimaging studies (extensively reviewed by [3; 9]).

In contrast, the neural activity of separate inputs to the MTLC is highly overlapping and therefore allows processing the shared structure of these separate inputs (representational bindings). For example, the first presentation of an item in a particular context, e.g. during encoding, weakly activates a large number of MTLC neurons, whereas repeated and thus familiar stimuli although in a different context, e.g. during a recognition test, activate only a subset of these neurons representing the familiar stimuli but every neuron is activated to a stronger degree [32]. Thus, during recognition the presentation of a studied item initiates a set of processes that may be described in a more cognitive framework as a comparison between the neural activity associated with the short-lived 
representation of the actual stimulus and the confined activity in the MTLC of the previous encounter of that stimulus. As a result, a scalar familiarity signal is provided that tracks the global similarity between the test probe and the studied items [33]. It should be noted that similar to the information based accounts it is assumed that, due to the divergent neural connections of the MTLC sub regions to neocortical areas, different structures within the MTLC bind different features of the entire input [7, 34]. While the perirhinal cortex encodes information about objects, the parahippocampal cortex represents the respective context of that input (Figure 1C).

As a consequence of this representational binding the MTLC is capable extracting the general regularities inherent in the input over repeated exposures to that input. These regularities mainly comprise frequency of co-occurrence but may also include transition probabilities or temporal contingencies (e.g. red and green in a traffic light, or item positions in a list learning paradigm). However, there are limitations to the ability of the MTLC to abstract the regularities inherent in the recent input. As the MTLC receives the majority of its inputs from unimodal and polymodal association areas [34], representational bindings within the MTLC are necessarily based on superficial perceptual features. Consequently, the MTLC is hardly capable to create abstract representations that are essential for goal-directed behaviour. However, the prefrontal cortex (PFC) seems ideally suited for the abstraction of such behaviour-guiding representations [35]. Thus, while the MTLC mainly binds the representation of the actual item/context to the representation of a previous occurrence of that same item/context, the PFC mediates the binding of the actual event to a more abstract or prototypical representation of invariant and non-accidental features of that event.

This binding view is supported by recent studies demonstrating an impairment of patients with anterior MTL lesions, including the perirhinal cortex, in perceptual discrimination of complex objects with a large number of overlapping features [36]. More importantly, this impairment was largest for objects with pre-existing semantic representations, e.g. beasts as compared to novel objects such as bar codes. This is consistent with the present view, that representational bindings supported by the perirhinal cortex link the actual appearance of a particular object to the mental representation of previous experiences with that same object. In a similar vein, the parahippocampal cortex mediates representational bindings of contextual features. For instance, it was demonstrated that the parahippocampal cortex is more active for objects that are strongly associated with a specific context (e.g. roulette wheel) than for objects that are very weakly associated with many possible contexts (e.g. cherry) [37]. These examples underscore the important role of both cortices for representational binding by demonstrating that readdressing object and/or contextual features of object occurrence during the repeated processing of a particular event require the integrity/activity of perirhinal and parahippocampal cortex, respectively. 


\section{Summary}

The present chapter summarized part of the recent evidence for the role of the hippocampus and the surrounding MTLC in memory. Several accounts described hippocampal function in terms of the cognitive processes subserved by different substructures within the MTL others have focused more on the different information processed by these structures or on the underlying neural operations. Although accounts based on the dichotomy between recollection and familiarity have a long standing tradition in memory research, it is still an open question whether the brain actually operates on this dichotomy. The two other accounts are more directly related to the different structures and the respective neural processes. Despite the exact relation of the neural processes to the assumed cognitive processes remains to be clarified it seems that the neural processes account cuts across the boundaries inherent in the cognitive processes.

The list of accounts on hippocampal memory function is far from being complete. Other accounts implicate the hippocampus in recent but not in remote memories. These are special cases of the overarching issue of memory consolidation assuming that under certain circumstances memories can become independent of the hippocampus. Although these circumstances are subject to current debate they are of high importance for amnesia research. Yet others emphasize the role of the hippocampus in spatial memory. This will be covered in the next chapter. More general, these highly different views are suggestive of a more general role of MTL substructures in memory than discussed in the present chapter.

It should be also noted that the hippocampus and the perirhinal and parahippocampal cortices are interconnected with multiple brain areas in the parietal and frontal lobes. As the focus of this chapter was on the role of the hippocampus in memory the important contribution of these other brain structures was not covered. However, only when their role was taken into account a more complete picture of the importance of the medial temporal lobe for the formation of declarative memories can be drawn. 


\section{References:}

1. Scoville WB, Milner B: Loss of recent memory after bilateral hippocampal lesions. J Neurol Neurosurg Psychiatry 1957; 20:11-21.

2. Wixted JT, Squire LR: The medial temporal lobe and the attributes of memory. Trends in Cognitive Neuroscience 2011; 15(5):210-217.

3. Eichenbaum HB, Yonelinas AP, Ranganath $\mathrm{C}$ : The medial temporal lobe and recognition memory. Annu Rev Neurosci 2007; 30:123-152.

4. Squire LR, Wixted JT, Clark RE: Recognition memory and the medial temporal lobe: a new perspective. Nat Rev Neurosci 2007 Nov; 8(11):872-883.

5. Davachi L, Wagner AD: Hippocampal contributions to episodic encoding: insights from relational and item-based learning. Journal of Neurophysiology 2002; 88:982-990.

6. Diana RA, Yonelinas AP, Ranganath C: The effects of unitization on familiarity-based source memory: testing a behavioral prediction derived from neuroimaging data. J Exp Psychol Learn Mem Cogn 2008; 34(4):730-740.

7. Aggleton JP: Multiple anatomical systems embedded within the primate medial temporal lobe: Implications for hippocampal function. Neuroscience and Biobehavioral Reviews 2012; 36(7):1579-96.

8. Davachi L: Item, context and relational episodic encoding in humans. Curr Opin Neurobiol 2006; 16(6):693-700.

9. Diana RA, Yonelinas AP, Ranganath C: Imaging recollection and familiarity in the medial temporal lobe: a three-component model. Trends Cogn Sci 2007 Sep;11(9):379-386.

10. Opitz B: Neural binding mechanisms in learning and memory. Neuroscience and Biobehavioral Reviews 2010; 34(7):1036-46.

11. Brown MW, Aggleton JP: Recognition memory: what are the roles of the perirhinal cortex and hippocampus? Nat Rev Neurosci 2001 Jan; 2(1):51-61.

12. Yonelinas AP, Quamme JR, Widaman KF, Kroll NEA, Sauvéand MJ, Knight RT: Mild hypoxia disrupts recollection, not familiarity. Cogn Affect Behav Neurosci 2004; 4(3):393-400.

13. Aggleton JP, Vann SD, Denby C, Dix S, Mayes AR, Roberts N, Yonelinas AP: Sparing of the familiarity component of recognition memory in a patient with hippocampal pathology. Neuropsychologia 2005; 43(12):1810-1823.

14. Bowles B, Crupi C, Mirsattari SM, Pigott SE, Parrent AG, Pruessner JC, Yonelinas AP, Köhler S: Impaired familiarity with preserved recollection after anterior temporal-lobe resection that spares the hippocampus. Proceedings of the National Academy of Sciences of the United States of America 2007; 104(41):16382-7.

15. Fortin NJ, Wright SP, Eichenbaum H: Recollection-like memory retrieval in rats is dependent on the hippocampus. Nature 2004; 431(7005):188-91. 
16. Dobbins IG, Rice HJ, Wagner AD, Schacter DL: Memory orientation and success: separable neurocognitive components underlying episodic recognition. Neuropsychologia 2003; 41(3):318-333.

17. Yonelinas AP: The nature of recollection and familiarity: A review of 30 years of research. Journal of Memry and Language 2002; 46:441-517.

18. Montaldi D, Spencer TJ, Roberts N, Mayes AR: The neural system that mediates familiarity memory. Hippocampus 2006; 16(5):504-520.

19. Daselaar SM, Fleck MS, Cabeza R: Triple dissociation in the medial temporal lobes: recollection, familiarity, and novelty. J Neurophysiol 2006; 96(4):1902-1911.

20. Yassa MA, Stark CEL: Multiple signals of recognition memory in the medial temporal lobe. Hippocampus 2008; 18(9):945-954.

21. Holdstock JS, Mayes AR, Roberts N, Cezayirli E, Isaac CL, O'Reilly RC: Under what conditions is recognition spared relative to recall after selective hippocampal damage in humans? Hippocampus 2002; 12:341-351.

22. Kirwan CB, Stark CEL: Medial temporal lobe activation during encoding and retrieval of novel face-name pairs. Hippocampus 2004; 14(7):919-930.

23. Davachi L, Mitchell JP, Wagner AD: Multiple routes to memory: distinct medial temporal lobe processes build item and source memories. Proc Natl Acad Sci U S A 2003; 100(4):2157-2162.

24. Uncapher MR, Otten LJ, Rugg MD: Episodic encoding is more than the sum of its parts: an $\{f M R I\}$ investigation of multifeatural contextual encoding.s. Neuron 2006; 52(3):547-556.

25. Mayes AR, Holdstock JS, Isaac CL, Montaldi D, Grigor J, Gummer A, Cariga P, Downes JJ, Tsivilis D, Gaffan D, Gong Q, Norman KA: Associative recognition in a patient with selective hippocampal lesions and relatively normal item recognition. Hippocampus 2004; 14(6):763784.

26. Ryan JD, Althoff RR, Whitlow S, Cohen NJ: Amnesia Is a Deficit in Relational Memory. Psychol Sci 2000; 11:454-461.

27. Komorowski RW, Manns JR, Eichenbaum H: Robust conjunctive item-place coding by hippocampal neurons parallels learning what happens where. The Journal of Neuroscience 2009; 29(31):9918-29.

28. Wirth S, Yanike M, Frank LM, Smith AC, Brown EN, Suzuki WA: Single neurons in the hippocampus and learning ofnew associations. Science 2003; 300:1578-1581.

29. Ekstrom AD, Kahana MJ, Caplan JB, Fields TA, Isham EA, Newman EL, Fried I: Cellular networks underlying human spatial navigation. Nature 2003; 425:184-187.

30. Mayes AR, Montaldi D, Migo E: Associative memory and the medial temporal lobes. Trends Cogn Sci 2007; 11(3):126-135. 
31. Mayes AR, Holdstock JS, Isaac CL, Hunkin NM, Roberts N: Relative sparing of item recognition memory in a patient with adult-onset damage limited to the hippocampus. Hippocampus 2002; 12(3):325-340.

32. O'Reilly RC, Norman KA: Hippocampal and neocortical contributions to memory: advances in the complementary learning systems approach. Trends in Cognitive Sciences 2002; 6:505510.

33. Hintzman DL: Similarity, global matching, and judgments of frequency. Mem Cognit 2001; 29:547-556.

34. Suzuki WA, Amaral DG: Functional neuroanatomy of the medial temporal lobe memory system. Cortex 2004; 40(1):220-222.

35. Simons JS, Spiers HJ: Prefrontal and medial temporal lobe interactions in long-term memory. Nature Rev Neurosci 2003; 4:637-648.

36. Barense MD, Gaffan D, Graham KS: The human medial temporal lobe processes online representations of complex objects. Neuropsychologia 2007; 45(13):2963-2974.

37. Bar M, Aminoff E: Cortical Analysis of Visual Context. Neuron 2003; 38:258-347. 

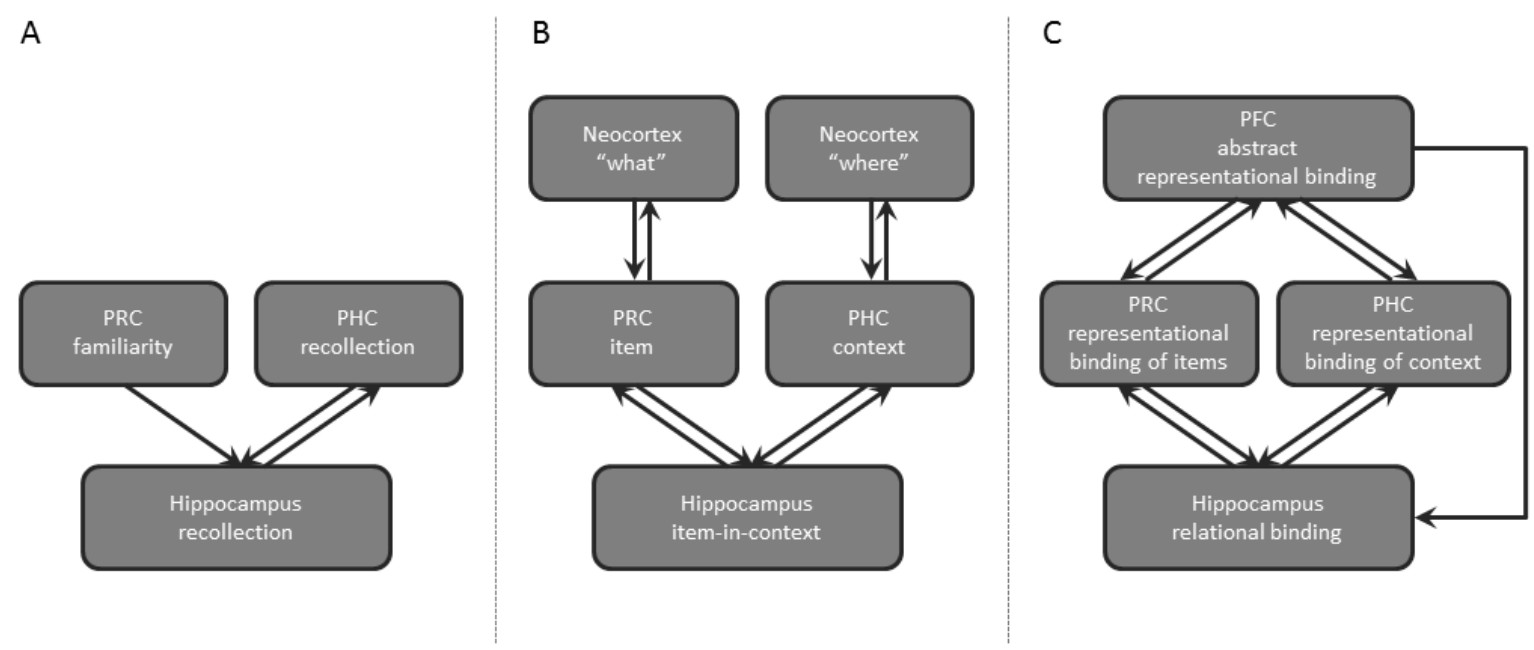

Figure 1. Schematic illustrations of the core assumptions of (A) Cognitive Processes Accounts, (B) Information Based Accounts (cf. [3]) and (C) Neural Processes Based Accounts (cf. [10]) about the role of different substructures within the medial temporal lobes in memory. PRC - perirhinal cortex, PHC - parahippocampal cortex, PFC - prefrontal cortex 